\title{
Detection of Angiostrongylus vasorum in Red Foxes (Vulpes vulpes) from Brandenburg, Germany
}

Vera Härtwig', Christoph Schulze², Dieter Barutzki³ ${ }^{3}$ Roland Schaper ${ }^{4}$, Arwid Daugschies', Viktor Dyachenko ${ }^{3}(\triangle)$

${ }^{1}$ Institute of Parasitology, Veterinary Faculty, University of Leipzig, 04103 Leipzig, Germany

2 Berlin-Brandenburg State Laboratory, 15236 Frankfurt (Oder), Germany

${ }^{3}$ Veterinary Laboratory Freiburg, 79108 Freiburg i. Br., Germany

${ }^{4}$ Bayer Animal Health GmbH, 51368 Leverkusen, Germany

\section{Corresponding author:}

\section{Dr. Viktor Dyachenko}

E-mail: v.dyachenko@labor-freiburg.de

\section{Abstract}

Angiostrongylus (A.) vasorum is a nematode that causes angiostrongylosis in domestic and wild canids. Red foxes (Vulpes vulpes) and raccoon dogs (Nyctereutes procyonoides) are suspected of providing a wildlife reservoir for A. vasorum infections in pet dogs. To obtain data on the occurrence of A.vasorum in wildlife, red fox and raccoon dog carcasses (hunted or found dead) were collected from January to September 2009 in the Federal State of Brandenburg, Germany. Lung tissue samples were subjected to DNA extraction and examined for the presence of A.vasorum DNA by means of real-time PCR. A.vasorum DNA was detected in 11 out of $122(9.0 \%)$ lungs of red foxes and in none of the lung samples of raccoon dogs. These data suggest that red foxes are a reservoir of A. vasorum infections for pet dogs in this area.

\section{Introduction}

Angiostrongylus (A.) vasorum is a metastrongylid nematode that parasitizes the pulmonary artery and, less often, the right heart of domestic and wild canids. Discovered in Toulouse, France, in 1853 by Serres and described systematically in 1866 by Baillet, A. vasorum is now endemic in several European countries (Bolt et al. 1994). Distribution is characterised by isolated endemic foci surrounded by regions with no or only sporadic occurrence (Bolt et al. 1994). Foci exist in France, Denmark, Cornwall, Wales and the south-east of England (Bolt et al. 1994; Willingham et al. 1996; Saeed et al. 2006; Morgan et al. 2005; Morgan et al. 2008). Expansion is, however, occurring from these foci into regions that were formerly not endemic, as seen in Great Britain, where new cases have evolved in dogs from Scotland (Helm 
et al. 2009) and northern England (Yamakawa et al. 2009) as well as in foxes from Scotland (Philbey and Delgado 2013). An important endemic focus outside of Europe is the Canadian province of Newfoundland-Labrador, where $56 \%$ of red foxes were found to be infected with A.vasorum of European origin (Jeffery et al. 2004; Jefferies et al. 2010).

A. vasorum needs gastropods (slugs and snails) as intermediate hosts to maintain its indirect life cycle. The common frog (Rana temporaria) can act as a paratenic and an intermediate host (Bolt et al. 1993). Domestic chickens (Gallus gallus domesticus) may also act as paratenic hosts (Mozzer and Lima 2015). After mating, adult female worms shed eggs, which are transported via the blood stream from the cardiopulmonary arteries into the pulmonary capillaries, where first-stage larvae (L1) hatch and penetrate the capillary and alveolar walls to the airways (Bolt et al. 1994). They are coughed up, swallowed and shed with the faeces. The prepatent period varies between 38 and 57 days (Bolt et al. 1994), in other studies between 28 and 108 days (Cury et al. 1996). The intermediate host gets infected by taking up firststage larvae from faeces of infected animals. Infection of the definitive host takes place by ingestion of an intermediate host in which L1 have developed to third-stage larvae (L3). Third-stage larvae penetrate the walls of the host's gastrointestinal tract and, after invasion of the abdominal visceral lymph nodes, develop into fifth-stage larvae (L5) which migrate via the portal vein, liver and posterior vena cava to the right heart and pulmonary arteries (Bolt et al. 1994).

Symptoms of angiostrongylosis range from subclinical to severe and A. vasorum infection may have a fatal outcome (Simpson and Neal 1982; Chapman et al. 2004; Traversa et al. 2008). In dogs, disease is characterised by coughing and dyspnoea, exercise intolerance and weight loss; neurological and gastrointestinal signs and haemorrhagic diathesis are further symptoms that may occur (Chapman et al. 2004; Koch and Willesen 2009; Helm et al. 2010).
Diagnosis of A. vasorum infection is challenging and includes different diagnostic approaches. The most common diagnostic method is Baermann's technique from faeces for the detection of L1. Intermittent shedding of larvae in faeces can lead to false negative results (Oliveira-Jùnior et al. 2006; Traversa and Guglielmini 2008). Other diagnostic tools have been developed, such as enzyme-linked immunosorbent assays (ELISA) for the detection of antibodies (Cury et al. 1996; Cury et al. 2002) or antigen (VerzbergerEpshtein et al. 2008) in blood and real-time PCR for the detection of DNA of L1 in blood or faeces (Jefferies et al. 2009; Jefferies et al. 2011). A combination of indirect ELISA and real-time PCR from blood improves the detection rate of A.vasorum infection (Jefferies et al. 2011). The transient presence of larvae and adults in the circulation that has been suggested, challenges all diagnostic methods (Jefferies et al. 2009). More recently other ELISA-based assays detecting A. vasorum antigen and antibodies have been utilised for large-scale regional and country-wide investigations based on larger numbers of samples (Schnyder et al. 2011; Schucan et al. 2012; Schnyder et al. 2013). A test for the serological detection of circulating antigen (Angio Detect ${ }^{\mathrm{TM}}$ Test, IDEXX Laboratories, Westbrook, Maine, USA) for rapid diagnosis is now also commercially available for use in veterinary clinics (Schnyder et al. 2014). Angiostrongylosis in dogs is considered an emerging disease in Europe (Morgan et al. 2005; Traversa and Guglielmini 2008; Koch and Willesen 2009; Helm et al. 2010). Studies from Germany indicate an increasing risk of infection for German dogs in recent years (Barutzki and Schaper 2003; Barutzki and Schaper 2009; Taubert et al. 2009; Barutzki and Schaper 2011; Barutzki 2013). However, information about the occurrence of A.vasorum in wildlife species in Germany is sparse, and little is known about $A$. vasorum infections in dogs in the eastern part of Germany.

The aim of this study was to collect information about A.vasorum prevalence in potential wildlife reservoirs. Therefore lung tissue samples of red foxes and raccoon dogs from the Havelland, 


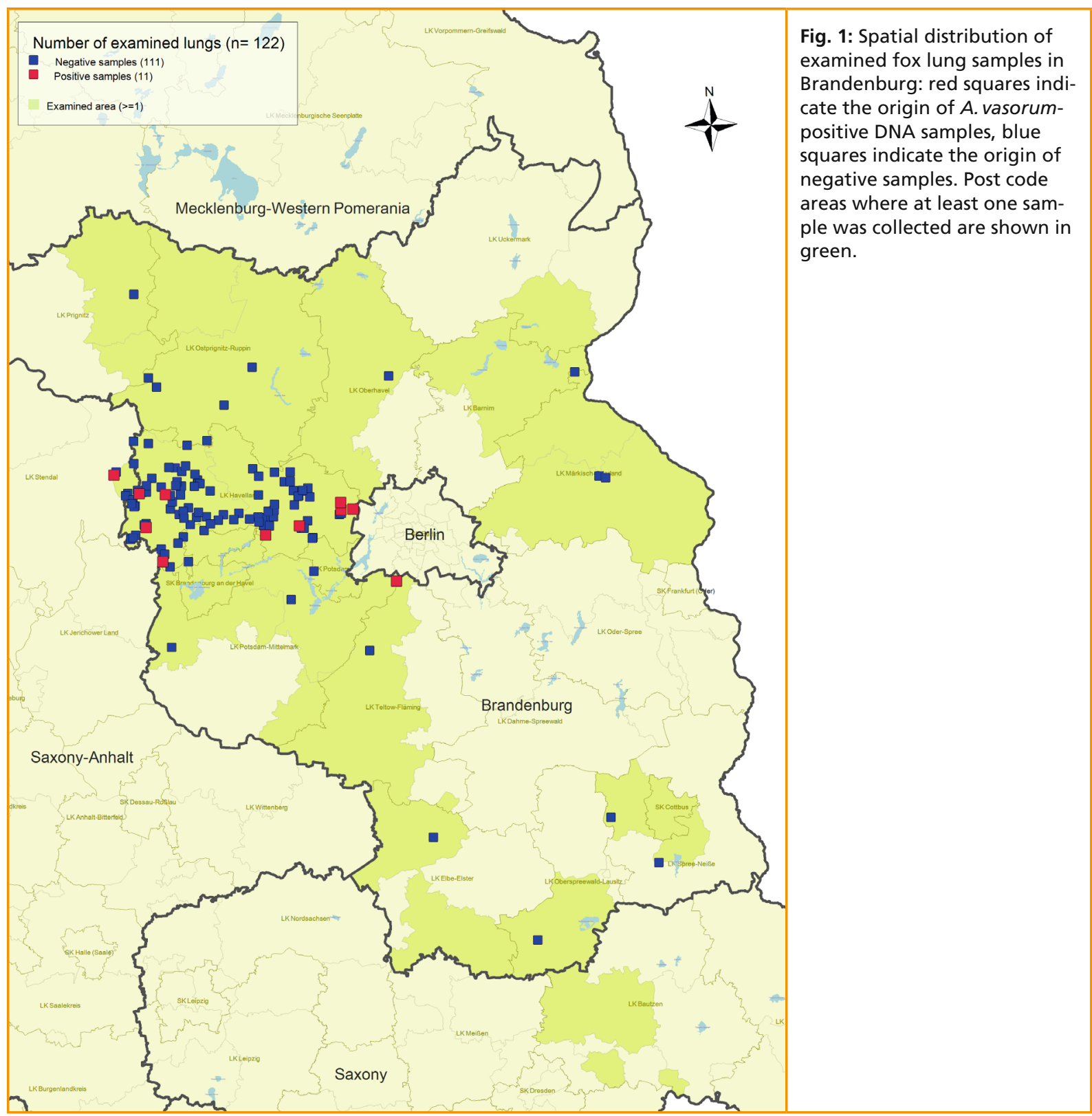

a region in the western part of the Federal State of Brandenburg, Germany, were examined for the presence of A. vasorum-specific DNA.

\section{Materials and methods}

\section{Sample collection and preparation}

A total of 122 red fox and 13 raccoon dog carcasses were collected from January to September 2009.
The animals were either found dead or were shot as part of the official rabies monitoring programme of the Federal State of Brandenburg, mainly in an area called the Havelland (Fig. 1). This area is rich in lakes and rivers, which provide an ideal harbour for intermediate and paratenic hosts. Carcasses were transported to the Berlin-Brandenburg State Laboratory in Frankfurt (Oder), numbered, allocated to specific geographical regions by post code and frozen for at least 7 days at $-80^{\circ} \mathrm{C}$ to inactivate 
Echinococcus multilocularis eggs. During necropsy, spleen and lung tissue was collected, frozen again at $-20^{\circ} \mathrm{C}$ and sent to the Institute of Parasitology, Veterinary Faculty of Leipzig, for further examination. Each lung sample was cut into small pieces and frozen in liquid nitrogen. The tissue was homogenised cryogenically with a prechilled mortar and pestle. After stirring the resulting lung powder thoroughly, $30 \mathrm{mg}$ was taken for DNA extraction. The mortar and pestle were cleaned with hot water and detergents, incubated with DNA AWAY (Roth, Germany) for a minute and finally disinfected with $70 \%$ alcohol after each lung sample.

\section{DNA extraction}

Total DNA was extracted using a QIAamp DNA Mini Kit (QIAGEN, Germany) following the manufacturer's instructions with some modifications: as $30 \mathrm{mg}$ tissue was used, the amount of buffer ATL, proteinase $\mathrm{K}$, buffer AL and absolute ethanol was doubled. DNA was eluted in $150 \mu \mathrm{l}$ of $\mathrm{AE}$ buffer. Extracted genomic DNA from each sample was analysed for degradation after electrophoresis in $0.8 \%$ agarose gel followed by ethidium bromide staining. In 116 out of 135 samples (85.9\%) non-degraded or partially degraded DNA (smear between 10,000 and $500 \mathrm{bp}$ ) was observed. In 19 samples (14.1\%) the DNA was degraded, presenting a smear below $700 \mathrm{bp}$. The DNA concentration was adjusted to $50 \mathrm{ng} / \mathrm{\mu l}$ in all samples prior to PCR amplification.

\section{Real-time PCR}

The presence of $A$. vasorum DNA was examined by means of real-time PCR targeting the ITS2 region. The PCR protocol was adapted from a previously described SYBR green real-time PCR assay (Jefferies et al. 2009). The reactions with a total of $125 \mathrm{ng}$ DNA in $25 \mu \mathrm{l}$ reaction volume were prepared with $12.5 \mu \mathrm{l}$ of Brilliant II Master Mix (Agilent Technologies, Germany) and $0.4 \mu \mathrm{M}$ of each primer and probe. Cycling reactions were carried out in a Stratagene Mx3000p cycler (Agilent Technologies, Germany) starting with an activation step at $95^{\circ} \mathrm{C}$ for $10 \mathrm{~min}$ followed by 50 cycles of $95^{\circ} \mathrm{C}$ for $15 \mathrm{~s}$ and $60^{\circ} \mathrm{C}$ for $1 \mathrm{~min}$. The following primers and probes were used: angio forward (GCGTGTGTTCATGTTTGGA), angio reverse (CATTACTAGCATACAAGCACATG) and angio probe (FAM-CGTCGTCGATATGCTACTGTTCCCG-TAMRA).

\section{Statistical analysis and geographic information system}

The confidence interval (95\% CI) of the prevalence found was calculated using the OpenEpi epidemiological calculator. The spatial distribution of all foxes and raccoon dogs included in this study was illustrated using the Regiograph software package (Fig. 1).

\section{Results}

In total, 11 out of 122 samples $(9.0 \% ; 95 \%$ CI $4.9-15.0 \%$ ) from red foxes showed an amplification of A.vasorum-specific DNA. In raccoon dogs, A. vasorum DNA was amplified in 0 out of 13 samples. All "no template controls" were negative. The FAM positive control (DNA, which was isolated from A. vasorum larvae) showed a Ct value of 39.13.

\section{Discussion}

The aim of this study was to determine the prevalence of A.vasorum infection in red foxes and raccoon dogs from the Havelland region, an area in the vicinity of the river Havel located in the western part of the Federal State of Brandenburg, Germany. A. vasorum has been diagnosed in German red foxes just recently, showing regionally differing prevalences of $8.4 \%, 19.1 \%$ and $27.3 \%$ for Thuringia, Hesse and Rhineland-Palatine, respectively (Schug et al. 2013). In our survey A. vasorum DNA was detected in $9.0 \%$ of lung samples of red foxes from the Federal State of Brandenburg. Indeed, our data are at the lower limit of the estimated range of prevalence compared to three other federal states in Germany. Two factors can affect estimated 
prevalence in this study: the results were generated by real-time PCR and therefore cannot be directly compared to those results generated by a classical examination procedure like autopsy; on the other hand there was an uneven distribution of fox carcasses since they were collected along the Havel river and thus give insight into the local region (Fig. 1). The molecular detection of A.vasorum we used in this work, including homogenisation of lung tissue in liquid nitrogen and DNA extraction has proven to be reliable earlier in a study for detection of Anaplasma phagocytophilum in red foxes and raccoon dogs (Härtwig et al. 2014). The overall prevalence of A. vasorum infections in German dogs is $0.5 \%$ as determined by examination of 24,667 faecal samples from healthy dogs and/ or dogs with gastrointestinal disorders (Barutzki and Schaper 2011). The rate of A.vasorum infection in dogs is generally lower than in foxes, as listed by Koch and Willesen (2009). In a preselected study on German dogs $(n=810)$ with clinical signs consistent with angiostrongylosis (respiratory/ cardiovascular symptoms, bleeding disorder and/ or neurological signs) $7.4 \%$ showed an infection with A.vasorum, diagnosed by faecal examination (Barutzki and Schaper 2009). Interestingly, most of the infected dogs originated from southern and western Germany (Barutzki and Schaper 2009), similar to the spatial distribution of canine A. vasorum infections in the study by Taubert et al. (2009). However, according to data from other countries A. vasorum seems to be expanding from endemic foci, as reviewed elsewhere (Morgan et al. 2005; Elsheikha et al. 2014). The estimated prevalence of $9.0 \%$ in red foxes from Brandenburg in this study suggests that the fox is a reservoir for A. vasorum infections for dogs in this region, which has not been recognised as an endemic area until now.

Data on A.vasorum infection in red foxes are not available from all neighbouring countries of Germany, but in Poland, the immediate neighbour to the Federal State of Brandenburg, a prevalence of $5.2 \%$ was determined in lungs and hearts from red foxes $(n=76)$ (Demiaszkiewiecz et al. 2014). In the
Netherlands A. vasorum infection was detected in $4.2 \%$ of examined red foxes $(\mathrm{n}=136)$. This nematode was one of 4 new helminth species found in Dutch foxes in the last 35 years (Franssen et al. 2014). The occurrence of helminth infections in red foxes from Denmark has been well studied, showing prevalences of A.vasorum of $48.6 \%(n=1040)$ in Northern Zealand, an endemic area within Denmark (Saeed et al. 2006). A recent study showed that A. vasorum is abundant in foxes $(\mathrm{n}=70)$ from the Copenhagen area with a prevalence of $80 \%$, but not in foxes $(\mathrm{n}=48)$ from Southern Jutland (Al-Sabi et al. 2014).

A lot of research is also done in the UK, which harbours some highly endemic foci and where A. vasorum has spread beyond traditional endemic areas (Blehaut et al. 2014; Kirk et al. 2014). The parasite is now widespread in central England, with clusters in the south-east of England and the south of Wales and a patchy distribution in northern England and Scotland. There is no longer an endemic focus in the south-west of England, as the authors found within a national questionnaire survey of 1419 veterinary practices (Kirk et al. 2014). After the first detection of A.vasorum in British foxes in Cornwall (Simpson 1996), 546 red foxes were studied by Morgan et al. (2008), showing an overall prevalence of $7.3 \%$ in the UK that varied from $0 \%$ in Scotland and northern England to $23.2 \%$ in south-east England. In addition to one report of A. vasorum in a captive fox in northern England in 1997 (Routh 2009), the parasite has been detected in 2 red fox cubs with neurological signs just recently in Scotland (Philbey and Delgado 2013). Disseminated angiostrongylosis was diagnosed in a red fox from central Italy, where $43.5 \%$ of foxes ( $\mathrm{n}=62$ ) were infected with A. vasorum (Eleni et al. 2014). In Spain, A. vasorum was found in $33.3 \%$ of red foxes $(n=48)$ from the Iberian peninsula; this is the only one of 10 species of wild carnivores to be infected with A.vasorum (Gerrikagoitia et al. 2010). Red foxes $(n=399)$ from the Iberian peninsula were investigated before, with a mean prevalence of $17.5 \%$ of A. vasorum infection varying in 
5 geographical areas from $3.8 \%$ in Andorra with a high-mountain climate to $36 \%$ on the Cantabrian coast with a maritime climate (Segovia et al. 2004). Climate seems to play an important role in parasite distribution, as also seen in Canada, where A. vasorum is only found in the south-eastern area of Newfoundland with a mean winter temperature above $-4^{\circ} \mathrm{C}$, whereas Crenosoma vulpis occurs island-wide (Jeffery et al. 2004).

Those two areas were used by Morgan et al. (2009) for a climate suitability prediction model for A.vasorum in Europe and the world, identifying areas with high, medium and low levels of A. vasorum presence. The climate dependency of A. vasorum is most likely due to the temperature and moisture sensitivity of the gastropod mollusc intermediate hosts of the parasite (Morgan et al. 2009). A moderate northward expansion of the predicted range is seen when running the model with a $2{ }^{\circ} \mathrm{C}$ global temperature increase (Morgan et al. 2009). Nevertheless, other factors in the distribution and expansion of the parasite also need to be considered, such as the growing fox population and transportation of dogs (Morgan et al. 2009; Helm et al. 2010; Elsheikha et al. 2014).

Although the raccoon dog has a wide range of parasites and a similar helminth spectrum to those of the red fox, A.vasorum has not been diagnosed in a raccoon dog so far (Thiess et al. 2001; Sutor et al. 2011; Bruzinskaite-Schmidhalter et al. 2012; Sutor et al. 2014). Thiess et al. (2001) investigated 74 raccoon dogs from East Brandenburg post mortem; 9.5\% showed an infection with Crenosoma vulpis and $1.4 \%$ with Capillaria aerophila but A. vasorum was not found in any of these animals. In Lithuania,
A. vasorum was not found in raccoon $\operatorname{dogs}(\mathrm{n}=99)$ post mortem (Bruzinskaite-Schmidhalter et al. 2012). In fact, a relatively low number of raccoon $\operatorname{dogs}(n=13)$ was included in our study. None of them showed an amplification of A.vasorum-specific DNA in lung tissue, confirming previous data. Differences in helminth diversity and prevalence in the two species may occur due to a variation in host susceptibility and food preferences/availability (Thiess et al. 2001; Al Sabi et al. 2013). Further investigations of this invasive species are needed to clarify its susceptibility to A. vasorum.

In conclusion, the A.vasorum prevalence of $9.0 \%$ in red foxes from Brandenburg in our study could be the first hint of a possible progressive or established expansion of this nematode into the northeast of Germany. Further extensive examinations of foxes and dogs from eastern Germany are necessary to verify the regional distribution of A.vasorum and to predict areas with a high infection risk.

\section{Ethicall Standards}

The study was performed in compliance with current national laws and regulations

\section{Funding}

The PCR part was funded by Bayer Animal Health $\mathrm{GmbH}$

\section{Conflict of interest}

Roland Schaper is current employee of Bayer Animal Health GmbH. All other authors declare no conflict of interest. 


\section{References}

Al-Sabi MNS, Chriel M, Jensen TH, Enemark HL (2013) Endoparasites of the raccoon dog (Nyctereutes procyonoides) and the red fox (Vulpes vulpes) in Denmark 2009-2012 - A comparative study. Int J Paras (Paras. \& Wildlife) 2:144-151

Al-Sabi MNS, Halasa T, Kapel CMO (2014) Infections with cardiopulmonary and intestinal helminths and sarcoptic mange in red foxes from two different localities in Denmark. Acta Parasitologica 59(1):98-107

Barutzki D, Schaper R (2003) Endoparasites in dogs and cats in Germany 1999-2002. Parasitol Res 90(3):149-150

Barutzki D, Schaper R (2009) Natural infections of Angiostrongylus vasorum und Crenosoma vulpis in dogs in Germany (2007-2009). Parasitol Res 105(1):39-48

Barutzki D, Schaper R (2011) Results of parasitological examinations of faecal samples from cats and dogs in Germany between 2003 and 2010. Parasitol Res 109(1):45-60

Barutzki D (2013) Nematodeninfektionen des Respirationstrakts bei Hunden in Deutschland. Tierärztliche Praxis Kleintiere 41:326-336

Blehaut TRW, Hardstaff JL, Chapman PS, Pfeiffer DU, Boag AK, Guitian FJ (2014) Spatial, demographic and clinical patterns of Angiostrongylus vasorum infection in the dog population of Southern England. Vet Rec 175:148

Bolt G, Monrad J, Frandsen F, Henriksen P, Dietz HM (1993) The common frog (Rana temporia) as a potential paratenic and intermediate host for Angiostrongylus vasorum. Parasitol Res 79:428-430

Bolt G, Monrad J, Koch J, Jensen AL (1994) Canine angiostrongylosis: a review. Vet Rec 135:447-452

Bruzinskaite-Schmidhalter R, Sarkunas M, Malakauskas A, Mathis A, Torgerson PR, Deplazes P (2012) Helminths of red foxes (Vulpes vulpes) and raccoon dogs (Nyctereutes procyonoides) in Lithuania. Parasitology 139:120-127

Chapman PS, Boag AK, Guitian J, Boswood A (2004) Angiostrongylus vasorum infection in 23 dogs (1999-2002). J Small Anim Pract 45:435-440

Cury MC, Lima WS, Vitor RWA (1996) Enzyme-linked immunosorbent assay (ELISA) for the diagnosis of Angiostrongylus vasorum (Baillet, 1866) infection in dogs. Rev Med Vet 147:525-530

Cury MC, Guimarães MP, Lima WS, Vitor RWA (2002) Western blot analysis of the humoral response of dogs experimentally infected with Angiostrongylus vasorum (Baillet, 1866). Vet Parasitol 106:83-87
Demiaszkiewicz AW, Pyziel AM, Kuligowska I, Lachowicz J (2014) The first report of Angiostrongylus vasorum (Nematoda; Metastrongyloidea) in Poland, in red foxes (Vulpes vulpes). Acta Parasitologica 59(4):758-762

Eleni C, Grifoni G, Di Egidio A, Meoli R, De Liberato C (2014) Pathological findings of Angiostrongylus vasorum infection in red foxes (Vulpes vulpes) from Central Italy, with the first report of a disseminated infection in this host species. Parasitol Res 113:1247-1250

Elsheikha HM, Holmes SA, Wright I, Morgan ER, Lacher DW (2014) Recent advances in the epidemiology, clinical and diagnostic features, and control of canine cardio-pulmonary angiostrongylosis. Vet Res 45:92

Franssen F, Nijsse R, Mulder J, Cremers H, Dam C, Takumi K, van der Giessen J (2014) Increase in number of helminth species from Dutch red foxes over a 35-year period. Parasit \& Vect 7:166

Gerrikagoitia X, Barral M, Juste RA (2010) Angiostrongylus species in wild carnivores in the Iberian Peninsula. Vet Parasitol 174:175-180

Härtwig V, von Loewenich FD, Schulze C, Straubinger RK, Daugschies A, Dyachenko V (2014) Detection of Anaplasma phagocytophilum in red foxes (Vulpes vulpes) and raccoon dogs (Nyctereutes procyonoides) from Brandenburg, Germany. TTBD 5(3): $277-280$

Helm J, Gilleard JS, Jackson M, Redman E, Bell R (2009) A case of canine Angiostrongylus vasorum in Scotland confirmed by PCR and sequence analysis. J Small Anim Pract $50: 255-259$

Helm JR, Morgan ER, Jackson MW, Wotton P, Bell R (2010) Canine angiostrongylosis: an emerging disease in Europe. J Vet Emerg Crit Care 20(1):98-109

Jefferies R, Morgan ER, Shaw SE (2009) A SYBR green real-time PCR assay for the detection of the nematode Angiostrongylus vasorum in definitive and intermediate hosts. Vet Parasitol 166:112-118

Jefferies R, Shaw SE, Willesen J, Viney ME, Morgan ER (2010) Elucidating the spread of the emerging canid nematode Angiostrongylus vasorum between Palaearctic and Nearctic ecozones. Infect Genet Evol 10:561-568

Jefferies R, Morgan ER, Helm J, Robinson M, Shaw SE (2011) Improved detection of canine Angiostrongylus vasorum infection using real-time PCR and indirect ELISA. Parasitol Res 109:1577-1583

Jeffery RA, Lankester MW, McGrath MJ, Whitney HG (2004) Angiostrongylus vasorum and Crenosoma vulpis in red foxes (Vulpes vulpes) in Newfoundland, Canada. Can J Zool 82:66-74 
Kirk L, Limon G, Guitian FJ, Hermosilla C, Fox MT (2014) Angiostrongylus vasorum in Great Britain: a nationwide postal questionnaire survey of veterinary practices. Vet Rec 175:118

Koch J, Willesen JL (2009) Canine pulmonary angiostrongylosis: an update. Vet J 179:348-359

Morgan ER, Shaw SE, Brennan SF, De Waal TD, Jones BR, Mulcahy G (2005) Angiostrongylus vasorum: a real heartbreaker. Trends Parasitol 21:49-51

Morgan ER, Tomlinson A, Hunter S, Nichols T, Roberts E, Fox MT, Taylor MA (2008) Angiostrongylus vasorum and Eucolaeus aerophilus in foxes (Vulpes vulpes) in Great Britain. Vet Parasitol 154:48-57

Morgan ER, Jefferies R, Krajewski M, Ward P, Shaw SE (2009) Canine pulmonary angiostrongylosis: The influence of climate on parasite distribution. Parasitol Int 58:406-410

Mozzer LR, Lima WS (2015) Gallus gallus domesticus: Paratenic host of Angiostrongylus vasorum. Vet Parasitol $207: 81-84$

Oliveira-Júnior SD, Barcante JMP, Barcante TA, Dias SRC, Lima WS (2006) Larval output of infected and re-infected dogs with Angiostrongylus vasorum (Baillet, 1866) Kamensky, 1905. Vet Parasitol 141:101-106

Philbey AW, Delgado D (2013) Detection of Angiostrongylus vasorum in red foxes in Scotland. Vet Rec 173:148

Routh A (2009) Angiostrongylus vasorum infection in a fox in northern England. Vet Rec 602-603

Saeed I, Maddox-Hyttel C, Monrad J, Kapel CMO (2006) Helminths of red foxes (Vulpes vulpes) in Denmark. Vet Parasitol 139:168-179

Schnyder M, Tanner I, Webster P, Barutzki D, Deplazes P (2011) An ELISA for sensitive and specific detection of circulating antigen of Angiostrongylus vasorum in serum samples of naturally and experimentally infected dogs. Vet Parasitol 179:152-158

Schnyder M, Schaper R, Bilbrough G, Morgan ER, Deplazes P (2013) Seroepidemiological survey for canine angiostrongylosis in dogs from Germany and the UK using combined detection of Angiostrongylus vasorum antigen and specific antibodies. Parasitology 140(11):1442-1450

Schnyder M, Stebler K, Naucke TJ, Lorentz S, Deplazes P (2014) Evaluation of a rapid device for serological in-clinic diagnosis of canine Angiostrongylosis. Paras \& Vect 7: 72

Schucan A, Schnyder M, Tanner I, Barutzki D, Traversa D, Deplazes P (2012) Detection of specific antibodies in dogs infected with Angiostrongylus vasorum. Vet Parasitol 185:216-224
Schug K, Hermosilla C, Hirzmann J, Schaper R, Taubert A (2013) Endoparasitenfauna beim Rotfuchs in Deutschland unter besonderer Berücksichtigung von Angiostrongylus vasorum. Tagung DVG-Fachgruppe "Parasitologie und Parasitäre Erkrankungen” Gießen, 8.-10.July 2013:79-80

Segovia JM, Torres J, Miquel J (2004) Helminth parasites of the red fox (Vulpes vulpes L., 1758) in the Iberian Peninsula: an ecological study. Acta Parasitologica 49(1):67-79

Simpson VR, Neal C (1982) Angiostrongylus vasorum infection in dogs and slugs. Vet Rec 111:303-304

Simpson VR (1996) Angiostrongylus vasorum infection in foxes (Vulpes vulpes) in Cornwall. Vet Rec 139:443-445

Sutor A, Schwarz S, Conraths FJ (2011) Der Marderhund (Nyctereutes procyonoides) in Deutschland- ein etablierter Neubürger als Wirt und Vektor für Parasiten und andere Pathogene. BMTW 124:457-464

Sutor A, Schwarz S, Conraths FJ (2014) The biological potential of the raccoon dog (Nyctereutes procyonoides, Gray 1834) as an invasive species in Europe- new risks for disease spread? Acta Theriol 59:49-59

Taubert A, Pantchev N, Vrhovec MG, Bauer C, Hermosilla C (2009) Lungworm infections (Angiostrongylus vasorum, Crenosoma vulpis, Aelurostrongylus abstrusus) in dogs and cats in Germany and Denmark in 2003-2007. Vet Parasitol 159:175-180.

Thiess A, Schuster R, Nöckler K, Mix H (2001) Helminthenfunde beim einheimischen Marderhund Nyctereutes procyonoides (Gray, 1834). BMTW 114:273-276

Traversa D, Torbidone A, Malatesta D, Guglielmini C (2008) Occurrence of fatal canine Angiostrongylus vasorum infection in Italy. Vet Parasitol 152:162-166

Traversa D, Guglielmini C (2008) Feline aelurostrongylosis and canine angiostrongylosis: A challenging diagnosis for two emerging verminous pneumonia infections. Vet Parasitol 157:163-174

Verzberger-Epshtein I, Markham RJF, Sheppard JA, Stryhn H, Whitney H, Conboy GA (2008) Serologic detection of Angiostrongylus vasorum infection in dogs. Vet Parasitol 151: $53-60$

Willingham AL, Ockens NW, Kapel CMO, Monrad J (1996) A helminthological survey of wild red foxes (Vulpes vulpes) from the metropolitan area of Copenhagen. J Helminthol $70: 259-263$

Yamakawa Y, McGarry JW, Denk D, Dukes-McEwan J, Macdonald N, Mas A, McConnell F, Tatton B, Valentine EG, Wayne J, Williams JM, Hetzel U (2009) Emerging canine angiostrongylosis in northern England: five fatal cases. Vet Rec 164:149-152. 